\title{
Clonage du gène responsable de la maladie de Menkes
}

La première description des données cliniques, neurologiques et génétiques de cette maladie a été rapportée en 1962 par Menkes [1]. La maladie de Menkes est caractérisée par une dégénérescence neurologique associée à un retard mental, un état particulier des cheveux qui sont dépigmentés et crêpelés, un déficit des parois vasculaires et une évolution rapide vers une mort précoce (cn général avant l'âge de 5 ans). L'hypothèse d'unc anomalie du métabolisme du cuivre n'a été suggéréc que dix ans plus tard par Danks sur la base d'une ressemblance entrc lcs cheveux crêpelés des enfants atteints dc la maladie de Menkes et la laine de moutons élevés en Australie sur des sols de faible teneur en cuivre [2]. Cette hypothèse a été rapidement consolidée par l'observation de déficits en enzymes qui utilisent les ions cuivre comme cofacteurs et par l'établissement de corrélations entre ces déficits enzymatiques et les signes cliniques de la maladie de Menkes [3] (Tableau I). Des études biochimiques récentes utilisant des cultures de fibroblastes de patients atteints de la maladie de Menkes ont montré une accumulation intracellulaire du cuivre dans tous les tissus, sauf dans le foie et les villosités choriales, et ont suggéré une anomalie du transport intracellulaire du cuivre [4].

Utilisant des stratégies différentes de génétique inverse, fondées dans tous les cas sur la caractérisation cytogénétique et moléculaire de points de cassure en $\mathrm{Xq13.3}$ de deux réarrangements chromosomiques observés chez deux patients atteints de la maladie de Menkes [5], trois groupes indépendants [6-8] ont récemment isolé le gène responsable de cette maladie.

Il s'agit d'un gène constitué d'au moins 14 exons répartis sur un locus génomique en Xq13.3 d'au moins $120 \mathrm{~kb}$ (Chelly et al., résultats non publiés). L'ARNm d'environ $8,5 \mathrm{~kb}$ est exprimé dans tous les tissus (sauf dans le foie où le nivcau d'expression est plus faible). L'investigation du gène et de son transcrit chez les paticnts a révélé des délétions non chevauchantes du gène dans $16 \%$ des cas [7] et des anomalies quantitatives et/ou qualitatives de l'ARNm dans $70 \%$ des cas [6].

La séquence nucléotidique de l'ADNc a montré que la partic codante n'cst que de $4,5 \mathrm{~kb}$ (52\% de l'ARNm) ct a permis de prédire que le transcrit code pour une protéine de 1500 acides aminés [6]. L'analysc assistéc par ordinateur de la séquence peptidique a suggéré que le produit du gènc responsable de la maladie de Menkes est un transporteur de cation, membre de la famille des ATPases de type P (figure 1). La comparaison de la séquence peptidique à celles des banques de données a montré une très forte homologie avec l'ATPase transporteur du cuivre $\left(\mathrm{Cu}^{2+}\right)$ d'Enterococcus hirae et des homologies significatives avec d'autres transportcurs de cations de procaryotes et d'cucaryotes. I.a protéine du gènc de Menkes scrait formée d'un domainc $\mathrm{N}$-terminal, vraiscmblablcment de liaison au cuivre, contenant six répétitions du motif Gly-Met-ThrCys-X-X-Cys similaircs à ceux des

Tableau I

ENZYMES QUI UTILISENT LES IONS Cu ${ }^{2}+$ COMME COFACTEURS ET SIGNES CLINIQUES OBSERVÉS EN CAS DE DÉFICIT

\begin{tabular}{|c|c|c|}
\hline Enzyme & Fonction & Conséquences cliniques \\
\hline Cytochrome c oxydase & $\begin{array}{l}\text { Transport d'électron } \\
\text { (mitochondrie) }\end{array}$ & $\begin{array}{l}\text { Faiblesse musculaire, } \\
\text { troubles visuels, ataxie }\end{array}$ \\
\hline Superoxyde dismutase & $\begin{array}{l}\text { Détoxification des } \\
\text { radicaux libres }\end{array}$ & $\begin{array}{l}\text { Dégénérescence myélini- } \\
\text { que et spasticité }\end{array}$ \\
\hline Dopamine $\beta$-hydroxylase & $\begin{array}{l}\text { Production de caté- } \\
\text { cholamines }\end{array}$ & $\begin{array}{l}\text { Hypothermie, anorexie, } \\
\text { somnolence, déshydrata- } \\
\text { tion, troubles respirato- } \\
\text { res, hypotension, ataxie }\end{array}$ \\
\hline Lysyl oxydase & $\begin{array}{l}\text { Liaison entre molé- } \\
\text { cules de collagène }\end{array}$ & $\begin{array}{l}\text { Anomalies du tissu con- } \\
\text { jonctif : complications } \\
\text { vasculaires, diverticules, } \\
\text { peau et articulations } \\
\text { flasques }\end{array}$ \\
\hline Tyrosinase & $\begin{array}{l}\text { Production de méla- } \\
\text { nine }\end{array}$ & Dépigmentation \\
\hline Enzyme à identifier & $\begin{array}{l}\text { Liaison entre molé- } \\
\text { cules de kératine }\end{array}$ & $\begin{array}{l}\text { Cheveux crêpelés, eczé- } \\
\text { ma }\end{array}$ \\
\hline
\end{tabular}




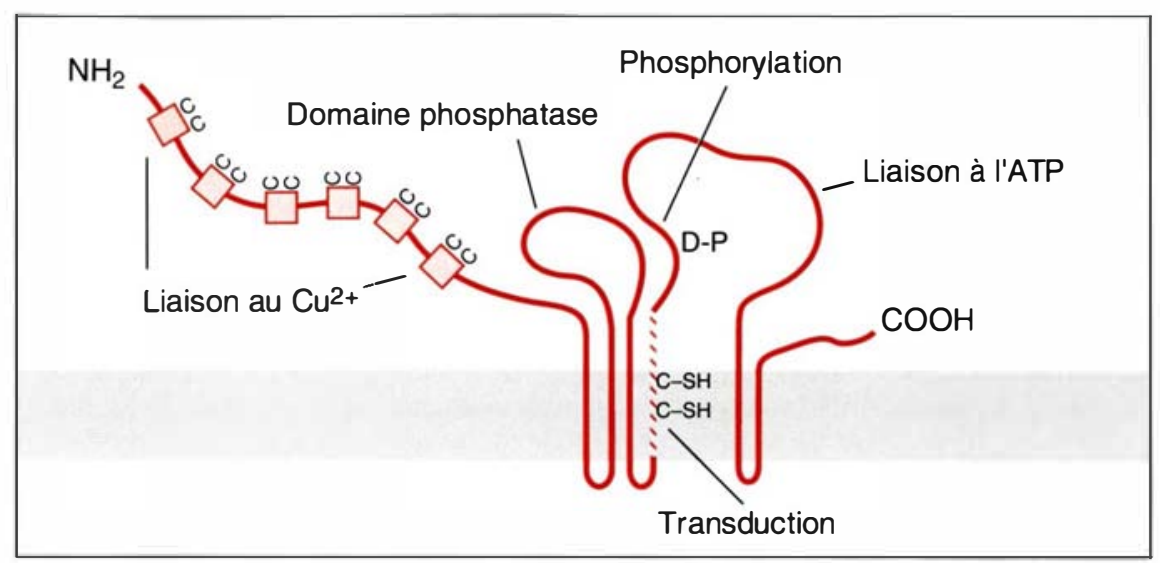

Figure 1. Structure prédite de la protéine produite par le gène responsable de la maladie de Menkes, une ATPase de type P [6]. Les boites avec les résidus cystéine $(C)$ représentent les motifs potentiels de liaison au cuivre. D-P : correspond au résidu acide aspartique en position 1044 qui subit la phosphorylation au cours du transport du cation cuivre. Le domaine phosphatase adjacent catalyserait l'hydrolyse de ce phosphate au cours du cycle réactif associé au transport du $\mathrm{Cu}^{2+}$.

\begin{tabular}{|c|c|c|c|c|c|c|}
\hline \multirow[t]{2}{*}{$\mathrm{NH}_{2}$} & $\begin{array}{c}100 \\
\perp\end{array}$ & $\stackrel{200}{1}$ & $\stackrel{300}{\perp}$ & $\begin{array}{c}400 \\
1\end{array}$ & $\begin{array}{c}500 \\
\perp \\
\end{array}$ & $\begin{array}{c}600 \\
\perp \\
\end{array}$ \\
\hline & $\Gamma$ LML1 & $\Gamma \mathrm{LML2}$ & $\Gamma$ LML3 & $\Gamma$ LML4 & $\lceil$ LML5 & $\Gamma$ LML6 \\
\hline \multicolumn{7}{|l|}{$\mathbf{8}$} \\
\hline \multicolumn{2}{|c|}{ MNK-LML1 } & \multicolumn{5}{|c|}{ VEGMTCNSCVWTIEQQIGKVNGVHHI KVSLEEKNATI I } \\
\hline \multicolumn{2}{|c|}{ MNK-LML2 } & \multicolumn{5}{|c|}{ VEGMTCHSCT ST IEGKI GKLQGVQR I KV SLDNQEATI } \\
\hline \multicolumn{2}{|c|}{$\begin{array}{l}\text { MNK-LML3 } \\
\text { MNK-LML4 }\end{array}$} & \multicolumn{5}{|c|}{ 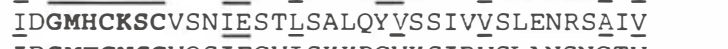 } \\
\hline \multicolumn{2}{|c|}{ MNK-LML4 } & \multicolumn{5}{|c|}{ IDGMTCNSCVQSIEGVISKKPGVKSIRVSSLANSNGTV } \\
\hline \multicolumn{2}{|c|}{$\begin{array}{l}\text { MNK-LML5 } \\
\text { MNK-LML6 }\end{array}$} & \multicolumn{5}{|c|}{$\underline{V}$ TGMTCASCVANIERNLRREEGIY YSILVALMAGKAEE } \\
\hline \multicolumn{2}{|c|}{ MNK - LML6 } & \multicolumn{5}{|c|}{ 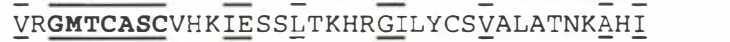 } \\
\hline \multicolumn{2}{|c|}{$\begin{array}{l}\mathrm{Hg}++, \operatorname{merP} \\
\mathrm{Hg++} \text { merA }\end{array}$} & \multicolumn{5}{|c|}{ 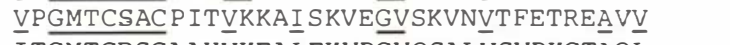 } \\
\hline \multicolumn{2}{|c|}{$\mathrm{Hg}++, \operatorname{merA}$} & \multicolumn{5}{|c|}{$\bar{I} T \overline{G M T C D S C} A A H \bar{V} K E A \bar{L} E K V P \overline{G V} Q S A L \bar{V} S V P K G T \bar{A} Q \overline{\underline{L}}$} \\
\hline \multicolumn{2}{|c|}{$\mathrm{Cd}_{++}, \mathrm{s}$. aureus } & \multicolumn{5}{|c|}{ 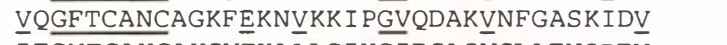 } \\
\hline \multicolumn{2}{|c|}{ HP1, T.ferrooxidans } & \multicolumn{5}{|c|}{ ITTMTCAHCAHSVEKA } \\
\hline \multicolumn{2}{|c|}{ FIXI, R.meliloti } & \multicolumn{5}{|c|}{ 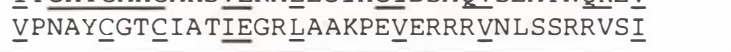 } \\
\hline
\end{tabular}

Figure 2. Domaine potentiel de liaison au cuivre de la protéine codée par le gène responsable de la maladie de Menkes. A. Position des motifs peptidiques conservés, GMTCXSC, dans la partie $\mathrm{NH}_{2}$-terminale de la protéine. Ces motifs correspondent vraisemblablement aux domaines de liaison des métaux lourds (LML). B. Alignement des six répétitions de 37 acides aminés de la protéine du gène de Menkes avec les séquences peptidiques de différents opérons bactériens de résistance aux métaux lourds [6-8]. En plus de la conservation du motif GMTCXXC, I'alignement montre la conservation de nombreux autres acides aminés (résidus soulignés).

$\mathrm{m} / \mathrm{s} n^{\circ} 3$ vol. 9, mars 93 opérons de résistance aux métaux lourds, tels que le $\mathrm{Cd}^{2+}$ et $\mathrm{Hg}^{2+} \mathrm{dc}$ certaines souches bactériennes (figure 2), de domaines de phosphorylation et de déphosphorylation, d'un domainc de liaison à l'ATP, typique des ATPases dc type $\mathrm{P}$, et de six domaines transmembranaircs correspondant au canalcation.

Les donnécs de séquence montrent donc quc lc gène responsable de la maladie de Menkes code pour une protéine impliquée dans le transport du cuivrc et suggèrent que les déficits en cnzymes qui utilisent les ions $\mathrm{Cu}^{2+}$ comme cofacteurs, et qui sont responsables des troubles cliniques, résultent d'un défaut d'incorporation du cuivrc au niveau des apo-enzymes. Ce mécanisme physiopathologique devrait être étayé par les études fonctionnelles et immunocytochimiques en cours.

Le taux trc̀s faible d'ARNm dans le foic adulte normal [7] et l'absence apparente de trouble de transport du cuivre dans le foie en cas de maladie de Mcnkes d'unc part, suggèrent, que cettc fonction serait assurée par un transportcur du cuivrc spécifique du foic, et d'autre part, suggèrent l'hypothèsc que la maladic de Wilson, caractérisće par un troublc du métabolisme du cuivre limité au foie, serait due à un défaut de cet hypothétique transportcur. Par ailleurs, l'isolement de ce gènc a permis de confirmer que le syndrome d'Ehler-Danlos lié à l'X, caractérisé par des anomalies limitées au tissu conjonctif, appelé aussi EhlerDanlos type IX ou cutis laxa lié à l'X [9], cst une forme allélique de la maladie de Menkes. En effet, Levinson et al. [10] ont montré une diminution drastique du taux du transcrit du gène de Menkes dans les fibroblastes en culturc de deux patients atteints de cutis laxa lié à l'X.

Le modèle animal équivalent à la maladie de Menkes est la mutation mottled de souris. L'isolement du gène responsable de cette mutation devrait faciliter la caractérisation des formes alléliques (on en connaît actuellement au moins 15) et fournir éventuellement des modèles pour le développement des applications thérapeutiques.

En conclusion, la caractérisation du gène responsable de la maladie de Menkes devrait faciliter de manière très significative le diagnostic, pratiqué 
auparavant par très peu de laboratoires et fondé sur l'étude de la cinétique d'accumulation $\mathrm{du}{ }^{64} \mathrm{Cu}^{2+}$ dans les fibroblastes en culture, et ouvrir de nouvclles perspectives pour l'étude de l'homéostasie cellulaire des métaux lourds essentiels

\section{Jamel Chelly}

Institute of Molecular Medicine, Human Genetics Laboratory, John Radcliffe Hospital, Headigton, Oxford OX3 9DU, RoyaumeUni.

\section{RÉFÉRENCES}

1. Menkes JH, Alter M, Stcigleder GK, Weakley DR, Sung JH. A sex-linked recessive disorder with retardation of growth, peculiar hair and focal cercbral and cellular degencration. P'ediatrics 1962; 29 : 764-79.

2. Danks IDM, Stevens BJ, Campbell PE, et al. Menkes' kinky-hair syndromc. Lancet $1972 ; 1: 110$.

3. Horn N, Tonnesen $\mathrm{T}$, Tümer $\mathrm{Z}$. Menkes discasc : an X-linked ncurological disorder of the copper metabolism. Brain l'athol $1992 ; 2: 1-10$.

4. Herd SM, Camakaris J, Christofferson R, Wookcy P, Danks DM. Uptake and cfflux of copper-64 in Menkes'- discase and normal continuous lymphoid cell lincs. Biochem $J$ $1987 ; 247: 341-7$.

5. 'Tumer Z, Chelly J, 'Tommerup $N$, Ishikawa-Brush Y, 'Tonnesen 'T', Monaco AP, Horn N. Charactcrization of a $1.0 \mathrm{Mb}$ contig spanning two chromosome breakpoints related to the Menkes discasc. Hum Mol (ienet $1992 ; 1: 483-9$.

6. Vulpe C, Lcvinson B, Whitncy S, Packman S, Gitschier J. Isolation of a candidate gene for Menkes discasc and evidence that it cncodes a copper-transporting A'T'Pasc. Nature Genet 199.3 ; 3 : 7-13

7. Chelly J, 'Tümer Z, 'Tønnesen 'T, Pettersonn $\mathrm{A}$, Ishikawa-Brush $\mathrm{Y}$, Tommcrup $\mathrm{N}$, Horn N, Monaco AP. Isolation of a candidate gene for Menkes discase that encodes a potential heavy metal binding protcin. Nature Genet 1993 ; 3 : 14-9.

8. Mcrcer LFB, Livingston J, Hall B, Paynter JA, Begy C, Chandrasckharappa S, lockhart P, Grimes A, Bhave M, Sicmicniak D, Glover TW. Isolation of a partial candidate gene for Menkes discasc by positional cloning. Nature Genet 1993 ; 3 : 20-5.

9. Pcltonen L, Kuivanicmi $\mathrm{H}$, Palotic A, Horn N, Kaitila I, Kivirikko I. Alterations in copper and collagen metabolism in the Menkes syndrome and a new subtype of the Ehler-Danlos syndromc. Biochemistry 1983 ; 22 : 6156-63.

10. Levinson B, Gitschicr J, Vulpe C, Whitney S, Yang S, Packman S. Are X-linked cuti.s laxa and Menkes discasc allclic? Nature Genet 1993; 3: 6
Une journée Recherche dans le cadre d'EUROCANCER 93 se tiendra à Paris le 27 avril 1993, sous la présidence de $P$. Tambourin et $M$. Boiron, sur le thème " De la recherche oncologique à l'innovation thérapeutique " avec, entre autres, le patronage de I'INSERM.

\section{Programme}

- Introduction et présentation de la journée (P. Tambourin, Paris) ; • Génétique des antigènes de rejet tumoral : nouvelles perspectives pour l'immunothérapie spécifique (Th. Boon, Bruxelles) ; - Contrôle de l'expression génétique (M. Yaniv, Paris) ; - Oncogènes mutants : cibles pour la thérapie (N. Lemoine, Londres) ; - Le couple prolifération-différenciation (L. Degos et $H$. de Thé, Paris) ; - Nouvelles cibles et nouveaux concepts en chimiothérapie anti-cancéreuse (N.W. Lobbezoo, Amsterdam); - Chimioprévention des cancers (A. Costa Milan) ; • Modulation de la résistance multidrogue associée à la P-glycoprotéine des cellules tumorales (G. Atassi, Paris) ; - Chimio- et radioprotecteurs (M. Marty, Paris) ; - Transfert de gènes (Th. Velu, Bruxelles et T. Blankenstein, Berlin): - Anticorps monoclonaux humains (J. Banchereau, Lyon); - Conclusion (M. Boiron, Paris).

L'examen des posters et leur discussion auront lieu au moment de la pause-déjeuner.

3 posters seront sélectionnés pour présentation orale par le jury scientifique d'Eurocancer: P. Tambourin (Président), F. Calvo, H. Fridman (INSERM U.255), S. Gisselbrecht, C.-J. Larsen (INSERM U.301), A. Tavitian (INSERM U.301), A. Tavitian (INSERM U.248), Th. Tursz.

Le prix du meilleur poster sera remis en fin de journée par P. Tambourin et le comité scientifique.

Les actes de cette journée seront disponibles sur forme d'une co-édition INSERM/John Libbey Eurotext dans la collection des Colloques de I'INSERM. Pour renseignement complémentaire et inscription, s'adresser au secrétariat scientifique : M. Boiron, M. Marty, M.-C. Guédès, Centre Hayem, Hôpital SaintLouis, 1, avenue Vellefaux, 75010 Paris. Tél. : 42.06.32.60 ou 42.03.36.56 - Télécopie : 42.41.14.70.

Un symposium international sur le thème "Ochratoxicose humaine et pathologies associées en Afrique et dans les pays en voie de développement " se tiendra à Bordeaux du 3 au 7 juillet 1993 avec le soutien de I'INSERM, de I'UNEP (United Nation Environment Program) et de la Région Aquitaine.

II réunira les principaux spécialistes internationaux de l'ochratoxine $A$ (mycotoxine, contaminant alimentaire) (G. Dirheimer, H. Bartsch, M. Castegnaro, T. Kuiper-Goodman, P. Galtier, P. Bach, R. Plestina, B. Hald, P. Steyn, Y. Ueno, etc.) et des équipes de chercheurs ayant mis en évidence des maladies liées à l'ochratoxicose humaine et animale dans leurs pays respectifs (Afrique, Asie). Les objectifs principaux sont les suivants:

- savoir si la néphropathie endémique des Balkans, vraisemblablement due à

l'ochratoxine $A$, existe en dehors de cette aire géographique ;

- quelles sont les pathologies associées à l'ochratoxicose dans les pays en voie de développement, en comparaison avec ce qui est observé dans les Balkans ;

- comment expliquer la spécificité rénale de cette mycotoxicose (néphrotoxicité et tumeurs) ;

- quels sont les moyens de prévention et/ou de traitement de l'ochratoxicose ( 25 à $60 \%$ au moins des personnes sont OTA-positives en Europe) ;

- comment mettre en place un réseau Nord-Sud de collaboration sur ce sujet qui concerne aussi bien les pays industrialisés que les pays sous-développés.

Les actes paraîtront pour le symposium, sous forme d'une co-édition INSERM/John Libbey Eurotext dans la collection des Colloques de I'INSERM.

Pour tout renseignement et inscription, s'adresser avant le 28 février 1993 au Pr. E. E. Creppy, Laboratoire de Toxicologie, Université de Bordeaux II, 33076 Bordeaux cedex, «Tél. : 56.91.84.07" Télécopie : 56.91.14.16. 